\title{
Longitudinal assessment of utilities in patients with migraine: an analysis of erenumab randomized controlled trials
}

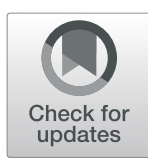

\author{
Gian Luca Di Tanna ${ }^{1,2^{*}}$ (D), Joshua K. Porter ${ }^{1}$, Richard B. Lipton ${ }^{3}$, Anthony J. Hatswell ${ }^{4}$, Sandhya Sapra ${ }^{5}$ and \\ Guillermo Villa ${ }^{1}$
}

\begin{abstract}
Background: Cost-effectiveness analyses in patients with migraine require estimates of patients' utility values and how these relate to monthly migraine days (MMDs). This analysis examined four different modelling approaches to assess utility values as a function of MMDs.

Methods: Disease-specific patient-reported outcomes from three erenumab clinical studies (two in episodic migraine [NCT02456740 and NCT02483585] and one in chronic migraine [NCT02066415]) were mapped to the 5-dimension EuroQol questionnaire (EQ-5D) as a function of the Migraine-Specific Quality of Life Questionnaire (MSQ) and the Headache Impact Test $\left(H I T-6^{T M}\right)$ using published algorithms. The mapped utility values were used to estimate generic, preference-based utility values suitable for use in economic models. Four models were assessed to explain utility values as a function of MMDs: a linear mixed effects model with restricted maximum likelihood (REML), a fractional response model with logit link, a fractional response model with probit link and a beta regression model.
\end{abstract}

Results: All models tested showed very similar fittings. Root mean squared errors were similar in the four models assessed $(0.115,0.114,0.114$ and 0.114 , for the linear mixed effect model with REML, fractional response model with logit link, fractional response model with probit link and beta regression model respectively), when mapped from MSQ. Mean absolute errors for the four models tested were also similar when mapped from MSQ $(0.085,0.086$, 0.085 and 0.085$)$ and HIT-6 and $(0.087,0.088,0.088$ and 0.089$)$ for the linear mixed effect model with REML, fractional response model with logit link, fractional response model with probit link and beta regression model, respectively.

Conclusions: This analysis describes the assessment of longitudinal approaches in modelling utility values and the four models proposed fitted the observed data well. Mapped utility values for patients treated with erenumab were generally higher than those for individuals treated with placebo with equivalent number of MMDs. Linking patient utility values to MMDs allows utility estimates for different levels of MMD to be predicted, for use in economic evaluations of preventive therapies.

Trial registration: ClinicalTrials.gov numbers of the trials used in this study: STRIVE, NCT02456740 (registered May 14, 2015), ARISE, NCT02483585 (registered June 12, 2015) and NCT02066415 (registered Feb 17, 2014).

Keywords: Utility, Migraine, Longitudinal, Modelling

\footnotetext{
* Correspondence: GDiTanna@georgeinstitute.org.au

${ }^{1}$ Economic Modeling Center of Excellence, Amgen (Europe) GmbH, Rotkreuz,

Switzerland

${ }^{2}$ The George Institute for Global Health, Newtown, New South Wales,

Australia

Full list of author information is available at the end of the article
}

(c) The Author(s). 2019 Open Access This article is distributed under the terms of the Creative Commons Attribution 4.0 International License (http://creativecommons.org/licenses/by/4.0/), which permits unrestricted use, distribution, and reproduction in any medium, provided you give appropriate credit to the original author(s) and the source, provide a link to the Creative Commons license, and indicate if changes were made. The Creative Commons Public Domain Dedication waiver (http://creativecommons.org/publicdomain/zero/1.0/) applies to the data made available in this article, unless otherwise stated. 


\section{Background}

Cost-effectiveness analyses are often used by reimbursement agencies to make decisions on whether to reimburse new healthcare interventions. Health-related quality of life (HRQoL) values can be expressed as utility scores, which capture social preferences for different health states [1]. Often, studies with HRQoL outcomes have repeated assessment over time and are longitudinal in nature. Analysing longitudinal data can present various challenges, such as missing data or the variations in patient HRQoL over time [2]. Therefore, it is important to consider the appropriate model when analysing longitudinal HRQoL data.

Regression models have been used to estimate treatment-effect impact on HRQoL [3]. Simple linear regression models, however, may not be optimal because health utility measures, including measures of HRQoL, may be multimodal and have ceiling effects, floor effects or skewed distributions [3-5]. In these circumstances, multivariable analyses, such as linear mixed models, may be more appropriate to estimate the changes in HRQoL over time. Linear mixed models, which are an extension of simple linear models and contain both fixed and random effects, can overcome the limitations associated with a longitudinal data set [2]. Recent analyses have demonstrated the suitability of the use of linear mixed models to measure HRQoL in longitudinal cohorts in a range of disease areas $[2,6]$. Anink et al. applied linear mixed models to examine HRQoL data in patients with juvenile idiopathic arthritis [6]. Wailoo et al. demonstrated the use of bespoke mixed models to model the 5-dimension EuroQol questionnaire (EQ-5D) in patients with ankylosing spondylitis [4]. Griffiths et al. estimated utility values from mixed regression models using EQ-5D data in patients with chronic heart failure [2].

According to the National Institute for Health and Care Excellence 'Guide to the Methods of Technology Appraisal', EQ-5D is the preferred method for measuring utilities [7, 8]. Utilities can be estimated from individual patient-level data collected as part of clinical studies (or extrapolated in the absence of long-term data) [9]. However, the collection of EQ$5 \mathrm{D}$ utilities is not always appropriate or possible in every disease state, so other methods may be used [7, 8]. Limited guidance exists on approaches to extrapolating outcomes such as utilities [10]. Applying existing algorithms is one of the options to derive utilities for health-state estimates when they are not available from the original data set $[10,11]$. Extrapolation methods should, however, consider processes that influence utilities that may not be linked to clinical events (e.g. past medical history of a patient or changes in clinical practice over time that may affect current practice) [10]. These considerations are particularly relevant to migraine, a chronic neurological disorder with episodic attacks of headache and an array of other symptoms [12]. Migraine is a debilitating disease in which utilities are typically measured via the Health Utilities Index (HUI) or the EQ-5D [13-16]. Migraine has considerable negative effects on a person's HRQoL, in addition to a high economic burden due to high direct costs (physician visits, emergency department visits, etc.) and indirect costs (lost work days, decreased productivity at work, etc.) [17]. Migraine can be divided into two categories based on the number of days on which patients have a headache in a 28-daymonth. Chronic migraine $(\mathrm{CM})$ is defined as experiencing $\geq 15$ monthly headache days (MHD) for $\geq 3$ or more months, 8 of which meet the criteria for migraine and/or respond to migraine-specific treatments [12]. Episodic migraine (EM) is defined as experiencing $\leq 14$ MHD [18-20].

Reduction in the frequency of monthly migraine days (MMD) is an important measure in the efficacy of migraine prophylaxis; however, there are limited data on the relationship between migraine frequency and health status [15]. Furthermore, patient-level data collected within the time frame of a clinical study often cover too short a duration to assess the likely costs and benefits that may yield over an individual's entire lifetime [10]. Preventive treatment can reduce the burden and disability associated with migraine [21]. Erenumab is a fully human monoclonal antibody that specifically blocks the calcitonin generelated peptide receptor complex [22] and has been shown to have a favourable safety and efficacy profile in phase 2 and phase 3 clinical studies [23-25]. In 2018, erenumab was approved by the US Food and Drug Administration for the prevention of migraine in adults [26].

The pivotal erenumab clinical studies included endpoints that recorded HRQoL data. This study aimed to leverage the HRQoL data from these studies to estimate patient utility values associated with specific levels of MMD. Various models for utilities in the longitudinal framework were compared using the observed utility data. Quantifying how the primary outcomes of the clinical studies, that is, MMDs, relate to utility values is important to inform cost-effectiveness analyses of preventive therapies such as erenumab [27].

\section{Methods}

\section{Data source}

The populations assessed in the models are the populations of three pivotal erenumab clinical studies 
[23, 24, 28]. In the phase 3 (NCT02456740) STRIVE (Study to Evaluate the Efficacy and Safety of Erenumab in Migraine Prevention), 955 patients with EM were enrolled. In the phase 3 (NCT02483585) ARISE (A phase 3, Randomized, double-blind, placebocontrolled Study to Evaluate the efficacy and safety of AMG 334 in migraine prevention), 577 patients with EM were enrolled and in the phase 2 study, 667 patients with CM were enrolled. The EM studies recruited individuals with $\leq 14$ MHD and 4-14 MMDs per 28 days and the $\mathrm{CM}$ study recruited individuals with $\geq 15$ HDs per 28 days and $>8 \mathrm{MD}$. To generalize the influence that MMD frequency has on patient utility values, the patient-reported outcomes for the placebo and erenumab $(70 \mathrm{mg}$ and $140 \mathrm{mg}$ ) arms of the three studies were combined to produce a complete migraine data set. Patient-level data were obtained for the participants in each study, with the following variables extracted for use in the analysis as the covariate set: participant identification (ID), study ID, age (continuous), sex (categorical), race (categorical), MMD at baseline (count), MMD (count) and treatment status (categorical). Covariates were selected based on known associations and clinical advice from experts in the field [29, 30]. Study-level effects were originally included in the hierarchical models, but as they demonstrated a negligible amount of variability between the studies, this layer was removed. As the objective of the analysis was to estimate patient utility based on MMD across the full migraine spectrum, combined models based on both EM and CM were fitted. Furthermore, the trials were comparable in terms of patients characteristics [31], therefore only patients level data were retained in the multilevel models presented here.

\section{Data description}

Patient utilities in the model were estimated as a function of MMD. For this analysis, MMD refers to the number of migraine days during a 28-day period. In the three studies, patients' HRQoL and daily functioning were collected in a monthly assessment, using the Headache Impact Test $\left(\right.$ HIT- $\left.6^{\mathrm{m}}\right)$ [32] and the Migraine-Specific Quality of Life Questionnaire (MSQ) [33]. The HIT-6 is designed to provide a global measure of adverse headache impact. Via a HRQoL questionnaire, the HIT-6 evaluates six content areas: pain, role functioning, social functioning, energy/fatigue, cognition and emotional distress [34]. The MSQ is a 14-item HRQoL questionnaire that measures three dimensions of functional status (role prevention, role restrictive and emotional function) specific to migraine $[33,34]$. Both the MSQ and the HIT- 6 have been shown to be valid and reliable tools for measuring the adverse impact of headache [32, 35]. Disease-specific patientreported outcomes from the studies were mapped to the EQ-5D using published algorithms. The mapping algorithms applied here have been previously published by Gillard et al., and these algorithms have been validated to support the analysis of onabotulinum toxin A (Botox ${ }^{\circ}$ ) trial data (see Online Resource: Additional file 2: Table S1) [34]. The size of the prediction error of the validated models was assessed using root mean squared error (RMSE) and mean absolute error (MAE).

In addition to the complete case analysis, a multivariate imputation by chained equations (MICE; fully conditional specification [FCS] algorithm) was performed with the assumption that data were missing at random. The MICE-FCS technique is a standard methodology for dealing with missing data and is also appropriate in the context of longitudinal data. The variables used in the imputation model were mapped MSQ, mapped HIT-6, treatment, baseline MMD, MMD, visit, age, sex and race. This imputation assessed the robustness of the results according to the presence of missing data and was constructed on a FCS [36] and based on 15 multiple imputed data sets [37].

\section{Utility regression models}

For this analysis, four models were assessed: (1) a linear mixed effects model with REML, (2) a fractional response model with logit link, (3) a fractional response model with probit link and (4) a beta regression model. Multilevel modelling approaches were chosen in order to take account of the longitudinal framework of the three trials, which included measurements collected from the same participants at repeated intervals over the course of the studies. These multilevel modelling approaches were used to enable the clustering of observations at the patient level.

In all models, the covariate set was examined. The mean predicted utilities by MMD (and by treatment status) were estimated with standard errors using the delta method [38]. Multilevel modelling techniques estimate the differences between individuals, acknowledging that measurements from the same person over time are much more likely to be correlated than measurements from different individuals [39].

In all four models the covariates included were as follows: treatment status (erenumab $70 \mathrm{mg}$ or $140 \mathrm{mg}$ vs placebo), age, sex (female vs male), race (black, Asian, other vs white), MMD at baseline, MMD at each visit and visit. The mean predicted utilities by MMD (and by treatment status) were estimated with standard errors using the delta method [38]. 


\section{Linear mixed effects model with REML}

A linear mixed effects model has been estimated with the REML method as a random-effects at the patient level, to estimate subject-specific effects and to provide distilled estimates of the specified covariates (the fixed component of the model) and estimates of the random variation according to the individuals [2, 40]. Acknowledging that a standard linear regression model (although hierarchical) is not well suited for an outcome that has a delimited unit interval such as utility values, which are typically characterized by a truncated support at both ends of the distribution (usually ranging between 0 and 1) and with heteroscedasticity (i.e. the variance of the residuals is not constant) as an integral part of such limited dependent variables [41], models fitted under the generalized linear model (GLM) framework have been shown to produce better estimates than those estimated by the linear model [42].

\section{Linear mixed effects model with REML}

$$
\begin{aligned}
E\left(U_{t i} \mid X\right)= & \beta_{0}+\overline{\beta_{1}} \text { Treatment }^{J}+\beta_{2} \text { BaselineMMD }_{i} \\
& +\beta_{3} \text { MMD }_{t i}+\beta_{4} \text { Age }_{i}+\beta_{5} \text { Female }_{i} \\
& +\overline{\beta_{6}} \text { Race }^{K}+\bar{\beta}_{7} \text { Visit }^{T}+u_{i}
\end{aligned}
$$

Where $t=0,4,8,12,16,20,24^{\mathrm{a}}$ weeks and $i=1,2, .$. , 2197 patients; $J$ is erenumab $70 \mathrm{mg}$, erenumab $140 \mathrm{mg}$ (vs placebo), $K$ is black, other (vs white).

${ }^{\mathrm{a}} 16-24$ weeks for EM studies only.

\section{Fractional response models with a logit link function or a probit link function}

Another valid strategy for handling proportions data in which zeros and ones may appear (as well as intermediate values) [43] is the fractional response model [44]. This model can be estimated via the GLM suite using the logit link function (i.e. the logit transformation of the response variable) or the probit link function [45]. Robust standard errors have been estimated allowing for clustering at individual participant level.

\section{Fractional response models with a logit link function or a probit link function}

$$
\begin{aligned}
E\left(U_{t i} \mid X\right)= & G\left(\overline{\beta_{1}} \text { Treatment }^{J}+\beta_{2} \text { BaselineMMD }_{i}\right. \\
& +{ }_{\beta_{3}} \text { MMD }_{t i}+\beta_{4} \text { Age }_{i}+\beta_{5} \text { Female }_{i} \\
& \left.+\overline{\beta_{6}} \text { Race }^{K}+\overline{\beta_{7}} \text { Visit }^{T}\right)
\end{aligned}
$$

where $G($.$) is a probit or logit function.$

\section{Beta regression model}

The fourth model fitted is a beta regression that is useful to model continuous, $0-1$ bounded and beta distributed outcomes. In the data set for this analysis, outcomes were constrained to have values higher than 0 and less than 1. Because some patients had a mapped utility (EQ5D) value of 1 , these values were decreased by $1.110^{\mathrm{e}-16}$, a marginal decrease to ensure minimal difference from the original values. As for the fractional response models, robust standard errors were estimated.

The density of the beta-distributed dependent variable $\mathrm{U}$ conditional on covariates $\mathrm{X}$ can be written as

$$
f\left(U ; \mu_{X}, \phi_{X}\right)=\frac{\Gamma\left(\phi_{X}\right)}{\Gamma\left(\mu_{X} \phi_{X}\right) \Gamma\left[\left(1-\mu_{X}\right) \phi_{X}\right]} U^{\mu_{X} \phi_{X}-1}(1-U)^{\left(1-\mu_{X}\right) \phi_{X}-1}
$$

Where $\mu_{X}=E\left(U_{t i} \mid X\right)$ is linked to the covariates set by $g\left(\mu_{X}\right)$ (a logit function of the linear predictor described above) and $\phi_{X}$ is the scale parameter of the conditional variance of $U$.

Goodness of fit of the regression models was assessed by RMSE, MAE and visual assessments.

All statistical analyses have been conducted using Stata 15 (StataCorp 2017 Stata Statistical Software, Release 15; StataCorp LLC, College Station, TX, USA).

\section{Results}

\section{Baseline characteristics}

The analysis sample included data from 2199 patients. Characteristics of the patients from the three studies are presented in Table 1. Baseline characteristics were similar across the three studies. For example, the average age was in the range 40.4-42.9 years across the three studies. The majority of patients in all studies were white and female, as is typical in migraine.

\section{Validated mapping algorithms}

In episodic migraine, the HIT-6 and MSQ algorithms explained 8 and $14 \%$ of the variance, respectively, as measured by adjusted $R^{2}$, and had similar prediction errors (RMSE of 0.32). In chronic migraine, the HIT-6 and MSQ algorithms explained 19 and $30 \%$ of the variance, respectively, and had similar prediction errors (RMSE of 0.33 and 0.32 ).

\section{Comparison of regression outputs and utility values}

Four regression models were fitted using mapped utility values, MMD and treatment group (erenumab $70 \mathrm{mg}, 140 \mathrm{mg}$ and placebo), adjusting for age, sex, race and baseline MMD in the various time periods considered. Mapped utility values are described in Table 2 and the distribution of the mapped utilities values are shown in Additional file 1: Figure S1 HIT-6 mapped mean (standard deviation [SD]) utility values were consistently higher than MSQ mapped mean (SD) utility values for patients treated with erenumab 
Table 1 Baseline characteristics of patients in the erenumab clinical trials [23, 24, 28]

\begin{tabular}{|c|c|c|c|c|c|c|c|c|}
\hline \multirow[t]{2}{*}{ Characteristic } & \multicolumn{3}{|c|}{$\begin{array}{l}\text { Episodic migraine } \\
\text { (NCT02456740) } \\
\text { STRIVE }\end{array}$} & \multicolumn{2}{|c|}{$\begin{array}{l}\text { Episodic migraine } \\
\text { (NCT02483585) } \\
\text { ARISE }\end{array}$} & \multicolumn{3}{|c|}{$\begin{array}{l}\text { Chronic migraine } \\
\text { (NCT02066415) }\end{array}$} \\
\hline & Placebo & $\begin{array}{l}\text { Erenumab } \\
70 \mathrm{mg}\end{array}$ & $\begin{array}{l}\text { Erenumab } \\
140 \mathrm{mg}\end{array}$ & Placebo & $\begin{array}{l}\text { Erenumab } \\
70 \mathrm{mg}\end{array}$ & Placebo & $\begin{array}{l}\text { Erenumab } \\
70 \mathrm{mg}\end{array}$ & $\begin{array}{l}\text { Erenumab } \\
140 \mathrm{mg}\end{array}$ \\
\hline Number of patients & 319 & 317 & 319 & 291 & 286 & 286 & 191 & 190 \\
\hline Mean age, years (SD) & $41.3(11.2)$ & $41.1(11.3)$ & $40.4(11.1)$ & $42.2(11.5)$ & $42.3(11.4)$ & $42.1(11.3)$ & $41.4(11.3)$ & $42.9(11.1)$ \\
\hline \multicolumn{9}{|l|}{ Sex, n (\%) } \\
\hline Male & $45(14.1)$ & $48(15.2)$ & $47(14.7)$ & $44(15.1)$ & $41(14.3)$ & $60(21.0)$ & $25(13.1)$ & $30(15.8)$ \\
\hline Female & $274(85.9)$ & $269(84.8)$ & $272(85.3)$ & $247(84.9)$ & $245(85.7)$ & $226(79.0)$ & $166(86.9)$ & $160(84.2)$ \\
\hline \multicolumn{9}{|l|}{ Race, n (\%) } \\
\hline White & $276(86.8)$ & $280(88.6)$ & $293(91.9)$ & $259(89.0)$ & $259(90.6)$ & $268(93.7)$ & $176(92.1)$ & $184(96.8)$ \\
\hline Black & $24(7.5)$ & $24(7.6)$ & $18(5.6)$ & $27(9.3)$ & $24(8.4)$ & $11(3.8)$ & $10(5.2)$ & $6(3.2)$ \\
\hline Other & $18(5.7)$ & $12(3.8)$ & $8(2.5)$ & $5(1.7)$ & $3(1.0)$ & $7(2.5)$ & $5(2.6)$ & $0(0.0)$ \\
\hline MMD & $8.2 \pm 2.5$ & $8.3 \pm 2.5$ & $8.3 \pm 2.5$ & $8.4 \pm 2.6$ & $8.1 \pm 2.7$ & $18.2 \pm 4.7$ & $17.9 \pm 4.4$ & $17.8 \pm 4.7$ \\
\hline
\end{tabular}

Abbreviations: ARISE A phase 3, Randomised, double-blind, placebo-controlled Study to Evaluate the efficacy and safety of AMG 334 in migraine prevention, MMD monthly migraine day, SD standard deviation, STRIVE Study to evaluate the efficacy and safety of erenumab in migraine prevention

$70 \mathrm{mg}$ and $140 \mathrm{mg}$, and for patients receiving placebo. HIT-6 and MSQ mapped mean (SD) utility values increased over time up to week 16 for patients treated with erenumab $70 \mathrm{mg}$ and $140 \mathrm{mg}$, and for patients treated with placebo. MSQ and HIT-6 mapped mean utility values were lowest in the 4 weeks before randomization to treatment or placebo (week 0). Patients at week 0 were predicted to have MSQ mapped mean (SD) utility values of $0.633(0.163)$ and 0.617 (0.176) for patients treated with erenumab $70 \mathrm{mg}$ and $140 \mathrm{mg}$, respectively, and $0.615(0.173)$ for patients receiving placebo (Table 2 ).

The predicted mean utility values by number of MMD are shown in Figs 1and 2 after mapping from MSQ and HIT-6, respectively. Mapped utility values for erenumab patients were consistently higher than for placebo patients with the same number of MMD. All models tested showed similar fittings and fit the observed data well (Figs 1 and 2). Because of the different likelihood functions used for the four regression models proposed in this analysis, the fittings could not be compared via Akaike Information Criterion (AIC) or Bayesian Information Criterion (BIC).

The regression outputs for the utility values after mapping from MSQ and HIT-6 are shown in Tables 3 and 4, respectively. In all models tested, the treatment effect of erenumab $140 \mathrm{mg}$ compared with placebo was significantly higher when mapped from HIT-6 (Table 4). Treatment with erenumab $140 \mathrm{mg}$ compared with placebo was also significantly higher in all models tested when mapped from MSQ (Table 3). Treatment with erenumab $70 \mathrm{mg}$ compared with placebo was not significant when mapped from MSQ or HIT-6 (Tables 3 and 4). Baseline MMD was significant in all models tested apart from the linear

Table 2 Mean utility (EQ-5D) values extrapolated from MSQ and HIT-6

\begin{tabular}{|c|c|c|c|c|c|c|}
\hline \multirow{2}{*}{$\begin{array}{l}\text { Week, } \\
\text { mean } \\
\text { (SD) }\end{array}$} & \multicolumn{2}{|l|}{ Placebo } & \multicolumn{2}{|c|}{ Erenumab $70 \mathrm{mg}$} & \multicolumn{2}{|c|}{ Erenumab 140 mg } \\
\hline & MSQ & HIT-6 & MSQ & HIT-6 & MSQ & HIT-6 \\
\hline 0 & $0.615(0.173)$ & $0.671(0.136)$ & $0.633(0.163)$ & $0.677(0.125)$ & $0.617(0.176)$ & $0.675(0.145)$ \\
\hline 4 & $0.675(0.161)$ & $0.721(0.135)$ & $0.716(0.142)$ & $0.747(0.125)$ & $0.716(0.154)$ & $0.761(0.147)$ \\
\hline 8 & $0.687(0.160)$ & $0.732(0.137)$ & $0.726(0.141)$ & $0.771(0.133)$ & $0.729(0.153)$ & $0.775(0.147)$ \\
\hline 12 & $0.687(0.160)$ & $0.734(0.139)$ & $0.730(0.146)$ & $0.776(0.134)$ & $0.737(0.144)$ & $0.787(0.145)$ \\
\hline 16 & $0.736(0.120)$ & $0.781(0.119)$ & $0.765(0.111)$ & $0.810(0.122)$ & $0.775(0.094)$ & $0.828(0.114)$ \\
\hline 20 & $0.731(0.127)$ & $0.786(0.128)$ & $0.771(0.100)$ & $0.813(0.121)$ & $0.776(0.093)$ & $0.824(0.114)$ \\
\hline 24 & $0.729(0.129)$ & $0.781(0.132)$ & $0.763(0.111)$ & $0.812(0.122)$ & $0.776(0.091)$ & $0.824(0.114)$ \\
\hline
\end{tabular}



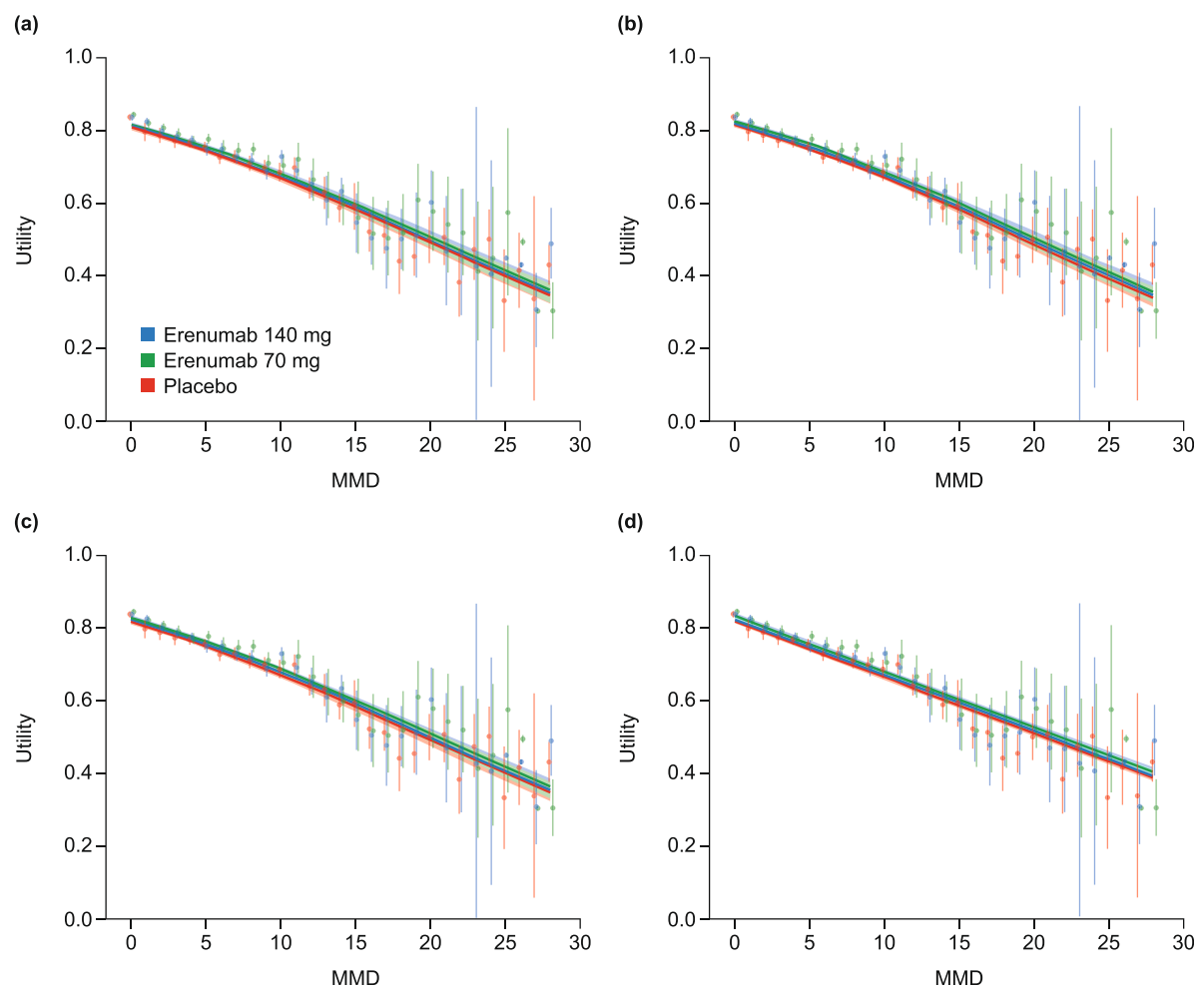

Fig. 1 Estimated EQ-5D mean utility values by MMD frequency for erenumab and placebo, mapped from MSQ. Abbreviations: Cl confidence interval, EQ-5D 5-dimension EuroQol questionnaire, FRM fractional response model, MMD monthly migraine day, MSQ Migraine-Specific Quality of Life Questionnaire, REML restricted maximum likelihood. Observed values with 95\% Cls are represented by blue, green and red vertical lines for erenumab $140 \mathrm{mg}$ and $70 \mathrm{mg}$ and placebo, respectively. (a) Linear mixed effects model with REML, (b) FRM (logit), (c) FRM (probit), (d) Beta regression model

model when mapped from HIT-6 (Table 3). RMSEs were similar in the four models assessed (0.115, $0.114,0.114$ and 0.114 for the mixed linear effects model with REML, fractional response model with logit link, fractional response model with probit link and beta regression model, respectively, when mapped from MSQ (Table 3). MAE for the four models tested were also similar when mapped from MSQ (0.086, $0.085,0.085$ and 0.085$)$ and HIT-6 and (0.087, 0.088, 0.088 and 0.089 ) for the mixed linear effects model with REML, fractional response model with logit link, fractional response model with probit link and beta regression model, respectively.

\section{Multiple imputation analyses}

The analyses based on the multiple imputed data sets were substantially similar to the complete case analyses for MSQ and HIT-6 (see Online Resource: Additional file 3: Table S2 and Additional file 4: Table S3). In the regression analyses, there were 10,977 and 10,971 complete case records, when mapped from MSQ and
HIT-6, respectively. There were similar proportions of missing observations in the multiple imputed data sets (200 [1.7\%] and 205 [1.8\%] when mapped from MSQ and HIT-6, respectively) compared with the complete case analysis (200 [7.5\%] and 205 [7.5\%] when mapped from MSQ and HIT-6, respectively).

Not all patients completed all the scheduled visits: 25 (3.7\%), 88 (9.2\%) and 30 (5.2\%) patients did not complete the planned assessments in the phase 2 study (which was planned for four visits), STRIVE (planned for seven visits) and ARISE (planned for four visits), respectively.

\section{Discussion}

Our analysis describes the assessment of longitudinal approaches in modelling utility values that go beyond simple linear models. In all cases, utility values decreased as the number of MMD increased, and these associations were non-linear with potential ceiling effects. The improvement in average utility values over time in the placebo groups is consistent 

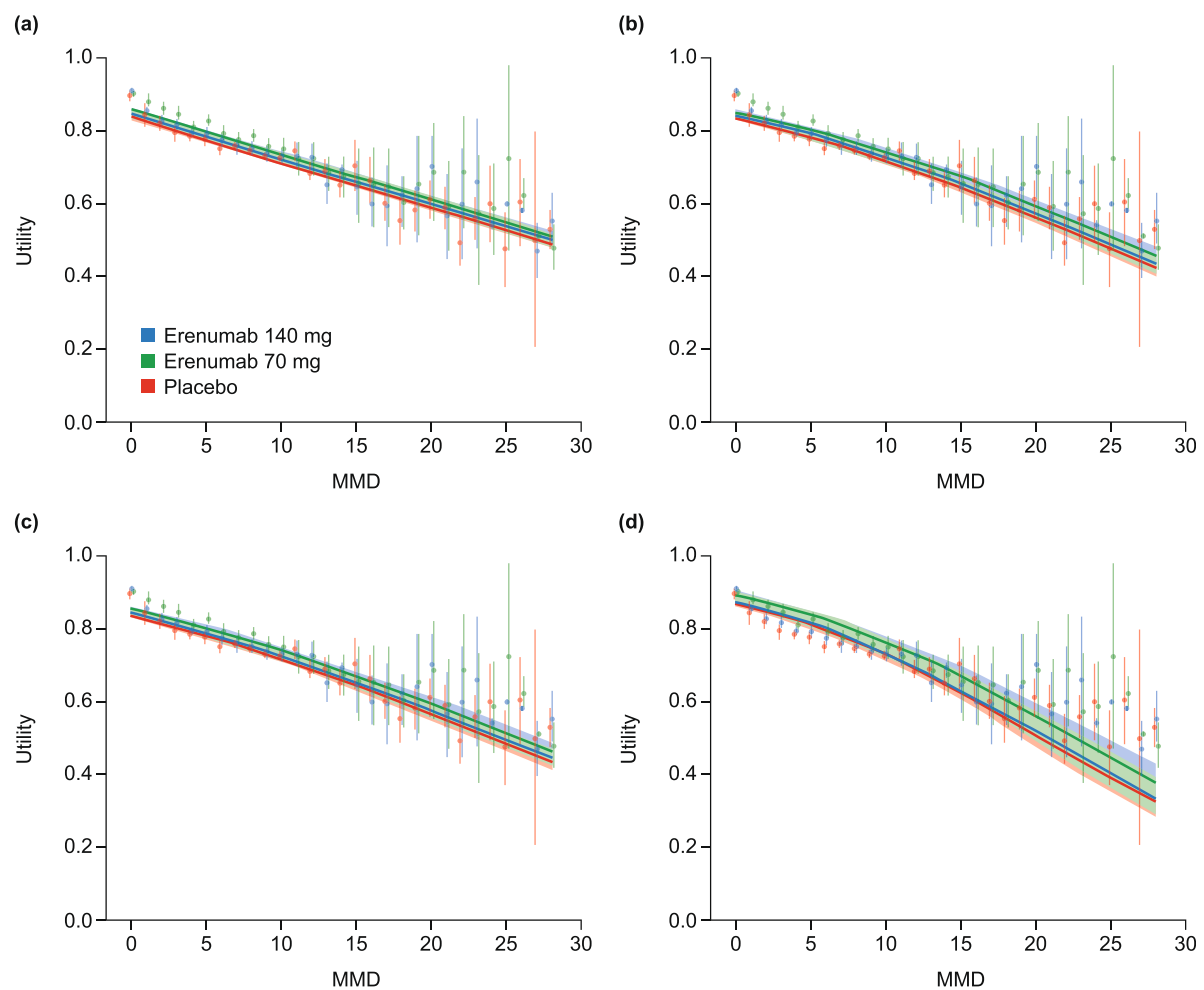

Fig. 2 Estimated EQ-5D mean utility values by MMD frequency for erenumab and placebo, mapped from HIT-6. Abbreviations: Cl confidence interval, EQ-5D 5-dimension EuroQol questionnaire, HIT-6 Headache Impact Test, MMD monthly migraine day, REML restricted maximum likelihood. Observed values with $95 \% \mathrm{Cls}$ are represented by blue, green and red vertical lines for erenumab $140 \mathrm{mg}$ and $70 \mathrm{mg}$ and placebo, respectively. (a) Linear mixed effects model with REML (b) Fractional response model with logit link, (c) Fractional response model with probit link and $(\mathbf{d})$ beta regression model

with the placebo effect on mean MMD frequency observed in the clinical studies [23, 24, 28]. Consistently, mapped utility values for patients treated with erenumab $70 \mathrm{mg}$ and $140 \mathrm{mg}$ were higher than those for participants treated with placebo with the same number of MMD, although only the $140 \mathrm{mg}$ dose of erenumab was significant. This finding is consistent with utility values applied in a previous economic model for onabotulinumtoxin $\mathrm{A}$, which assumed an additional treatment effect of active treatment compared with placebo [46]. This additional treatment effect is most likely driven by improvements in migraine duration and severity, that may not be fully captured by the primary clinical endpoint, MMD.

All models tested showed very similar fittings, although the beta regression model may be considered as the optimal candidate for longitudinal and bounded data, because the beta regression model has the flexibility of a beta distribution model and has previously been used to model quality-adjusted lifeyears in health economic studies [41, 47]. To determine the generalizability of this model, it would be necessary to examine mapped utility values from other study data.

Some limitations of the analysis have to be acknowledged. Firstly, the HIT-6 and MSQ scores were captured only monthly in the three clinical studies. It may be beneficial to capture HRQoL data more frequently to accurately capture patients' experiences within the 1 -month time periods [34, 48]. This is particularly relevant, because time and other factors can influence how individuals with migraine can report their HRQoL [34]. Secondly, the use of likelihood-based statistics such as AIC/BIC could not be used to compare models with different likelihood functions. The analysis is further limited by the duration of the erenumab clinical studies: longer studies may be able allow more robust models to be fitted. Finally, because there were very similar RMSEs between the models, it was important to assess the non-linear associations between utilities and MMD. In future studies, it would be useful to assess any longer-term time trends, introducing a specific fixed-effect covariate and assessing the potential 


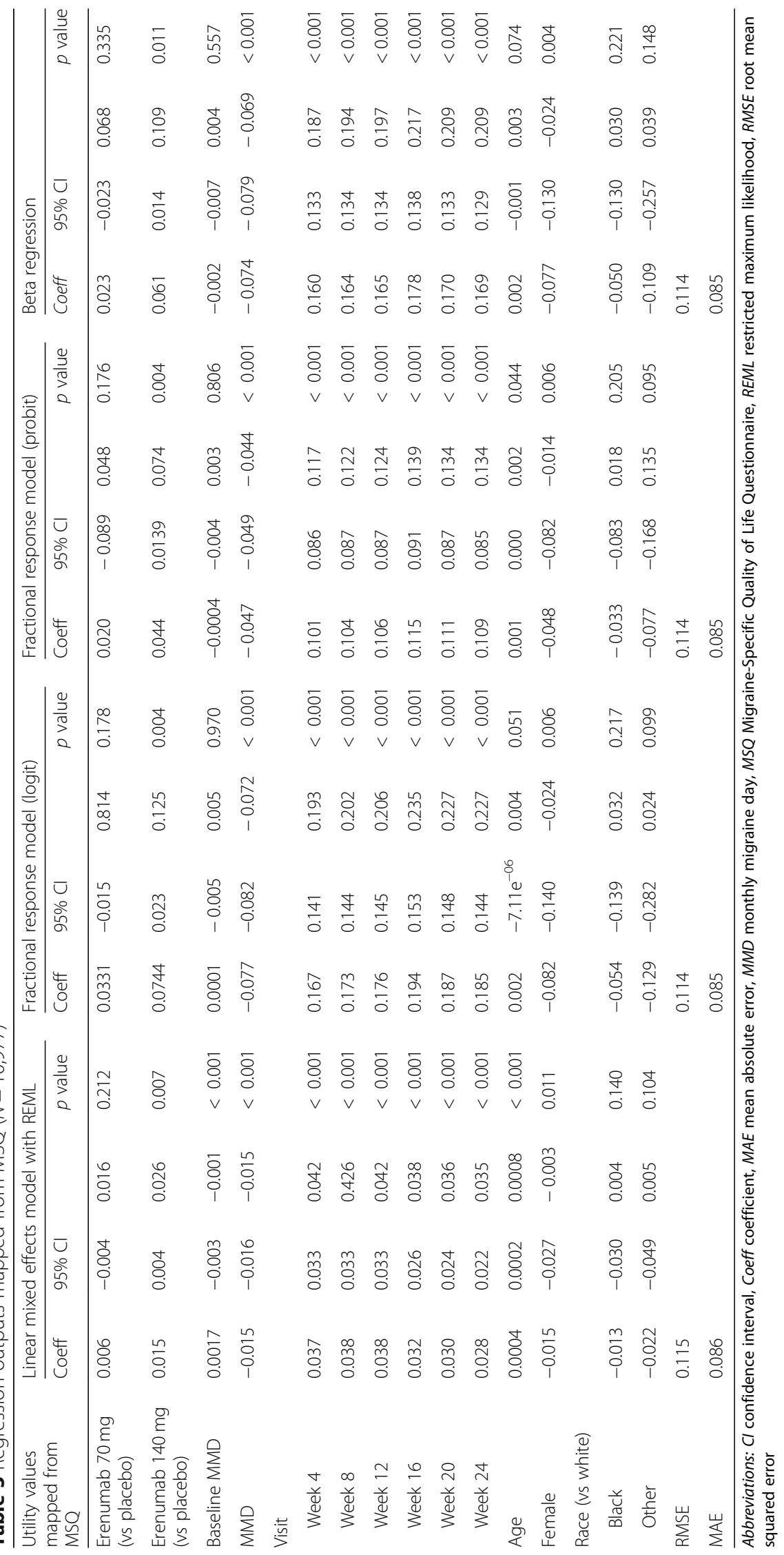




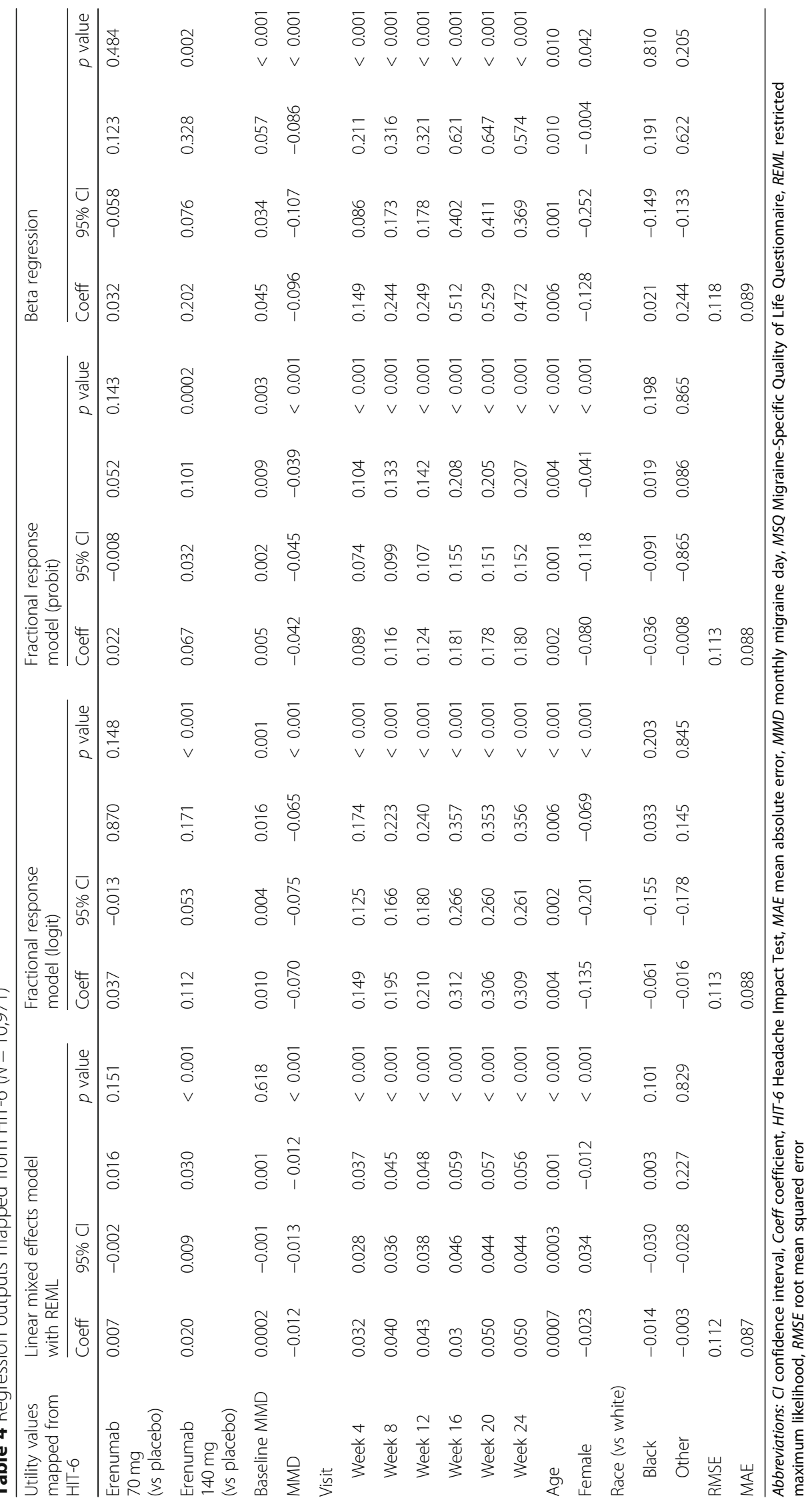


interaction between treatment and MMD. Exploration of such models as response mappings to predict the levels of utilities would be of interest. Future studies that examine mapping from a measure such as the Migraine Physical Function Impact Diary, which is a daily, migraine-specific measurement of patient-reported outcomes, would also be worth considering [48].

The analysis described here has applications for economic evaluations. Cost-utility analysis is widely recognized as a useful approach for measuring and comparing the efficiency of different health interventions [49]. Furthermore, longitudinal approaches for modelling utilities can be appropriate when considering economic evaluations because they can capture changes in health utility over time. In using utility values that are useful for decision-making bodies, the robust findings of this analysis, consistent across the models fitted, demonstrate the value of this data for health economic evaluations for migraine prevention and treatment.

\section{Conclusions}

Our analysis showed that all models fitted the observed data well. Mapped utility values for patients receiving erenumab were higher than those for patients with the same number of MMD receiving placebo, indicating that treating migraine may have benefit beyond simply reducing the number of migraines a patient experiences and may translate into improvements in HRQoL. Linking patient utility values to the number of MMD allows utility estimates for different levels of MMD to be predicted, for use in economic evaluations of preventive therapies. More broadly, the analysis demonstrates the application of different models for fitting utilities from study data.

\section{Supplementary information}

Supplementary information accompanies this paper at https://doi.org/10 1186/s12955-019-1242-6.

Additional file 1: Figure S1. Distribution of mapped utilities for MSQ and HIT-6. Abbreviations: EQ-5D 5-dimension EuroQol questionnaire, HIT-6 Headache Impact Test, MSQ Migraine-Specific Quality of life Questionnaire.

Additional file 2: Table S1. Mapping algorithm to estimate EQ-5D utility values from HIT-6 and MSQ [34]

Additional file 3: Table S2. Multiple imputation outputs for MSQ. Additional file 4: Table S3. Multiple imputation outputs for HIT-6.

\section{Abbreviations}

AIC: Akaike Information Criterion; ARISE: A phase 3, Randomized, doubleblind, placebo-controlled Study to Evaluate the efficacy and safety of AMG 334 in migraine prevention; BIC: Bayesian Information Criterion;

Cl: Confidence interval; CM: Chronic migraine; Coeff: Coefficient; EM: Episodic migraine; EQ-5D: 5 dimension EuroQol questionnaire; FCS: Fully conditional specification; FRM: Fractional response model; GLM: Generalized linear model; HD: Headache day; HIT-6: Headache Impact Test; HRQoL: Healthrelated quality of life; HUI: Health Utilities Index; MAE: Mean absolute error; MICE: Multivariate imputation by chained eqs.; MMD: Monthly migraine day; MSQ: Migraine-Specific Quality of Life Questionnaire; NCT: National clinical trial; REML: Restricted maximum likelihood; RMSE: Root mean squared error; SD: Standard deviation; STRIVE: Study to evaluate the efficacy and safety of erenumab in migraine prevention

\section{Acknowledgements}

We acknowledge medical writing support by Sinéad Flannery PhD of Oxford PharmaGenesis, Oxford, UK. Funding for this support was provided by Amgen (Europe) $\mathrm{GmbH}$.

\section{Authors' contributions}

All authors were involved in the conception and design of the study. GLDT, JP and GV were involved in the patient data collection and acquisition of the data. GLDT and JP were involved in analysis and interpretation of the data. All authors read and approved the final manuscript.

Funding

Funding for this work was provided by Amgen (Europe) $\mathrm{GmbH}$.

\section{Availability of data and materials}

Data Availability Statement.

Qualified researchers may request data from Amgen clinical studies. Complete details are available at the following: http://www.amgen.com/ datasharing

\section{Ethics approval and consent to participate}

The three trials used in this study were registered with ClinicalTrials.gov, numbers NCT02456740, NCT02483585 and NCT02066415 as previously reported. For each of the study sites the final study protocol was approved by an Institutional Review Board/Independent Ethics Committee. Informed consent was obtained from all individual participants included in the study.

\section{Consent for publication}

Not applicable.

\section{Competing interests}

GLDT was an employee of Amgen at the time of the study. RBL is the Edwin S. Lowe Professor of Neurology at the Albert Einstein College of Medicine in New York. He receives research support from the NIH: 2PO1 AG003949 (Program Director), 5 U10 NS077308 (PI), 1RO1 AG042595 (Investigator), RO1 NS082432 (Investigator), K23 NS09610 (Mentor), K23AG049466 (Mentor). He also receives support from the Migraine Research Foundation and the National Headache Foundation. He serves on the editorial board of Neurology and as senior advisor to Headache. He has reviewed for the National Institute on Aging and National Institute of Neurological Disorders and Stroke; holds stock options in eNeura Therapeutics and Biohaven Holdings; serves as consultant, advisory board member, or has received honoraria from: American Academy of Neurology, Alder, Allergan, American Headache Society, Amgen, Autonomic Technologies, Avanir, Biohaven, Biovision, Boston Scientific, Dr. Reddy's, Electrocore, Eli Lilly, eNeura Therapeutics, GlaxoSmithKline, Merck, Pernix, Pfizer, Supernus, Teva, Trigemina, Vector, Vedanta. He receives royalties from Wolff's Headache, 8th Edition, Oxford Press University, 2009, Wiley and Informa. AH was an employee of BresMed Health Solutions when the study was conducted, which has received consulting fees from Amgen. JP, SS and GV are employed by Amgen and have stock in Amgen.

\section{Author details}

${ }^{1}$ Economic Modeling Center of Excellence, Amgen (Europe) GmbH, Rotkreuz, Switzerland. ${ }^{2}$ The George Institute for Global Health, Newtown, New South Wales, Australia. ${ }^{3}$ Albert Einstein College of Medicine, Bronx, New York, NY, USA. ${ }^{4}$ Delta Hat Limited, Nottingham, UK. ${ }^{5}$ Amgen Inc., Thousand Oaks, CA, USA. 
Received: 16 November 2018 Accepted: 28 October 2019

Published online: 12 November 2019

\section{References}

1. Yeung MW, Tomlinson G, Loeb M, Sander B. Health-related quality of life in persons with West Nile virus infection: a longitudinal cohort study. Health Qual Life Outcomes. 2017;15(1):210.

2. Griffiths A, Paracha N, Davies A, Branscombe N, Cowie MR, Sculpher M. Analyzing health-related quality of life data to estimate parameters for costeffectiveness models: an example using longitudinal EQ-5D data from the SHIFT randomized controlled trial. Adv Ther. 2017:34(3):753-64.

3. Hunger M, Doring A, Holle R. Longitudinal beta regression models for analyzing health-related quality of life scores over time. BMC Med Res Methodol. 2012;12:144.

4. Wailoo A, Hernandez M, Philips C, Brophy S, Siebert S. Modeling health state utility values in ankylosing spondylitis: comparisons of direct and indirect methods. Value Health. 2015;18(4):425-31.

5. Kobelt G, Andlin-Sobocki P, Brophy S, Jonsson L, Calin A, Braun J. The burden of ankylosing spondylitis and the cost-effectiveness of treatment with infliximab (Remicade). Rheumatology. 2004;43(9):1158-66.

6. Anink J, Prince FH, Dijkstra M, Otten MH, Twilt M, ten Cate R, Gorter SL, et al. Long-term quality of life and functional outcome of patients with juvenile idiopathic arthritis in the biologic era: a longitudinal follow-up study in the Dutch Arthritis and Biologicals in Children Register. Rheumatology. 2015;54(11):1964-9.

7. Matza LS, Boye KS, Stewart KD, Curtis BH, Reaney M, Landrian AS. A qualitative examination of the content validity of the EQ-5D-5L in patients with type 2 diabetes. Health Qual Life Outcomes. 2015;13:192.

8. NICE (National Institute for Health and Care Excellence): Process and methods guides: NICE Process and Methods Guides. Guide to the Methods of Technology Appraisal 2013. London; 2013.

9. Wolowacz SE, Briggs A, Belozeroff V, Clarke P, Doward L, Goeree R, Lloyd A, et al. Estimating health-state utility for economic models in clinical studies: an ISPOR good research practices task force report. Value Health. 2016;19(6): 704-19.

10. Bojke L, Manca A, Asaria M, Mahon R, Ren S, Palmer S. How to appropriately extrapolate costs and Utilities in cost-effectiveness analysis. Pharmacoeconomics. 2017;35(8):767-76.

11. Loveman E, Jones J, Hartwell D, Bird A, Harris P, Welch K, Clegg A. The clinical effectiveness and cost-effectiveness of topotecan for small cell lung cancer: a systematic review and economic evaluation. Health Technol Assess. 2010;14(19):1-204.

12. Headache Classification Committee of the International Headache Society (IHS) The International Classification of Headache Disorders, 3rd edition. Cephalalgia. 2018;38(1):1-211.

13. Antonaci F, Nappi G, Galli F, Manzoni GC, Calabresi P, Costa A. Migraine and psychiatric comorbidity: a review of clinical findings. J Headache Pain. 2011; 12(2):115-25.

14. Leonardi M, Raggi A. Burden of migraine: international perspectives. Neurol Sci. 2013;34(Suppl 1):S117-8.

15. Brown JS, Neumann PJ, Papadopoulos G, Ruoff G, Diamond M, Menzin J. Migraine frequency and health utilities: findings from a multisite survey. Value Health. 2008;11(2):315-21.

16. Stafford MR, Hareendran A, Ng-Mak DS, Insinga RP, Xu R, Stull DE. EQ-5Dderived utility values for different levels of migraine severity from a UK sample of migraineurs. Health Qual Life Outcomes. 2012;10:65.

17. Munakata J, Hazard E, Serrano D, Klingman D, Rupnow MF, Tierce J, Reed M et al. Economic burden of transformed migraine: results from the American Migraine Prevalence and Prevention (AMPP) study. Headache. 2009:49(4): 498-508.

18. Headache Classification Subcommittee of the International Headache Society. The International Classification of Headache Disorders: 2nd edition. Cephalalgia. 2004:24(Suppl 1):9-160.

19. Katsarava Z, Buse DC, Manack AN, Lipton RB. Defining the differences between episodic migraine and chronic migraine. Curr Pain Headache Rep. 2012;16(1):86-92.

20. Lipton RB, Silberstein SD. Episodic and chronic migraine headache: breaking down barriers to optimal treatment and prevention. Headache. 2015; 55(Suppl 2):103-22 quiz 23-6.

21. Mennini FS, Gitto L, Martelletti P. Improving care through health economics analyses: cost of illness and headache. J Headache Pain. 2008;9(4):199-206.
22. Shi L, Lehto SG, Zhu DX, Sun H, Zhang J, Smith BP, Immke DC, et al. Pharmacologic characterization of AMG 334, a potent and selective human monoclonal antibody against the calcitonin gene-related peptide receptor. J Pharmacol Exp Ther. 2016;356(1):223-31.

23. Goadsby PJ, Reuter U, Hallstrom Y, Broessner G, Bonner JH, Zhang F, Sapra $\mathrm{S}$, et al. A controlled trial of erenumab for episodic migraine. N Engl J Med. 2017;377(22):2123-32

24. Tepper S, Ashina M, Reuter U, Brandes JL, Dolezil D, Silberstein S, Winner $P$, et al. Safety and efficacy of erenumab for preventive treatment of chronic migraine: a randomised, double-blind, placebo-controlled phase 2 trial. Lancet Neurol. 2017;16(6):425-34.

25. Sun H, Dodick DW, Silberstein S, Goadsby PJ, Reuter U, Ashina M, Saper J, et al. Safety and efficacy of AMG 334 for prevention of episodic migraine: a randomised, double-blind, placebo-controlled, phase 2 trial. Lancet Neurol. 2016;15(4):382-90.

26. Food and Drug Administration. 2018. Aimovig (erenumab). Prescribing Information [https://www.accessdata.fda.gov/drugsatfda_docs/label/2018/ 761077s000lbl.pdf ].

27. Di Tanna GLPJ, Lipton RB, Hatswell AJ, Sapra S, Villa G. Estimating patient utility in migraine: longitudinal analysis of erenumab clinical trial data. Value in Health. 2018;21:S225.

28. Dodick DW, Ashina M, Brandes JL, Kudrow D, Lanteri-Minet M, Osipova V, Palmer $\mathrm{K}$, et al. ARISE: a phase 3 randomized trial of erenumab for episodic migraine. Cephalalgia. 2018;38(6):1026-37.

29. Blumenfeld AM, Varon SF, Wilcox TK, Buse DC, Kawata AK, Manack A, Goadsby PJ, et al. Disability, HRQoL and resource use among chronic and episodic migraineurs: results from the international burden of migraine study (IBMS). Cephalalgia. 2011;31(3):301-15.

30. Bonafede M, Sapra S, Shah N, Tepper S, Cappell K, Desai P. Direct and indirect healthcare resource utilization and costs among migraine patients in the United States. Headache. 2018;58(5):700-14.

31. Porter JK, Di Tanna GL, Lipton RB, Sapra S, Villa G. Costs of acute headache medication use and productivity losses among patients with migraine: insights from three randomized controlled trials. Pharmacoecon Open. 2019; 3(3):411-7.

32. Yang M, Rendas-Baum $R$, Varon SF, Kosinski M. Validation of the headache impact test (HIT-6) across episodic and chronic migraine. Cephalalgia. 2011; 31(3):357-67.

33. Bagley CL, Rendas-Baum R, Maglinte GA, Yang M, Varon SF, Lee J, Kosinski M. Validating migraine-specific quality of life questionnaire $\mathrm{V} 2.1$ in episodic and chronic migraine. Headache. 2012;52(3):409-21.

34. Gillard PJ, Devine B, Varon SF, Liu L, Sullivan SD. Mapping from diseasespecific measures to health-state utility values in individuals with migraine. Value Health. 2012;15(3):485-94.

35. Martin BC, Pathak DS, Sharfman MI, Adelman JU, Taylor F, Kwong WJ, Jhingran P. Validity and reliability of the migraine-specific quality of life questionnaire (MSQ version 2.1). Headache. 2000;40(3):204-15.

36. Bartlett JW, Seaman SR, White IR, Carpenter JR. Alzheimer's disease neuroimaging I: multiple imputation of covariates by fully conditional specification: accommodating the substantive model. Stat Methods Med Res. 2015;24(4):462-87.

37. White IR, Royston P, Wood AM. Multiple imputation using chained equations: issues and guidance for practice. Stat Med. 2011;30(4):377-99.

38. Graubard BI, Korn EL. Predictive margins with survey data. Biometrics. 1999; 55(2):652-9.

39. Twisk J. Multilevel analysis in longitudinal studies. In: Applied Multilevel Analysis; 2006. p. p181.

40. Rabe-Hesketh S, Skrondal A. Multilevel and longitudinal modeling using Stata vol. In: II: Categorical Responses, Counts, and Survival, vol. II; 2012.

41. Basu A, Manca A. Regression estimators for generic health-related quality of life and quality-adjusted life years. Med Decis Mak. 2012;32(1):56-69.

42. Kieschnick R, McCullough BD. Regression analysis of variates observed on $(0,1)$ : percentages, proportions and fractions. Stat Model. 2003;3:193-213.

43. Baum C. Modeling proportions. Stata J. 2008;8(2):299-303.

44. Papke LWJ. Econometric methods for fractional response variables with an application to $401(\mathrm{k})$ plan participation rates. J Appl Econ. 1996;11(6):619-32.

45. Wooldridge JM. Fractional response models with endogeneous explanatory variables and heterogeneity; 2011.

46. Batty AJ, Hansen RN, Bloudek LM, Varon SF, Hayward EJ, Pennington BW, Lipton RB, et al. The cost-effectiveness of onabotulinumtoxinA for the 
prophylaxis of headache in adults with chronic migraine in the UK. J Med Econ. 2013;16(7):877-87.

47. Hubben GA, Bishai D, Pechlivanoglou P, Cattelan AM, Grisetti R, Facchin C, Compostella FA, et al. The societal burden of HIV/AIDS in northern Italy: an analysis of costs and quality of life. AIDS Care. 2008;20(4):449-55.

48. Kawata AK, Hsieh R, Bender R, Shaffer S, Revicki DA, Bayliss M, Buse DC, et al. Psychometric evaluation of a novel instrument assessing the impact of migraine on physical functioning: the migraine physical function impact diary. Headache. 2017;57(9):1385-98.

49. National Institute of Health and Care Excellence. The guidance manual.

\section{Publisher's Note}

Springer Nature remains neutral with regard to jurisdictional claims in published maps and institutional affiliations.

Ready to submit your research? Choose BMC and benefit from:

- fast, convenient online submission

- thorough peer review by experienced researchers in your field

- rapid publication on acceptance

- support for research data, including large and complex data types

- gold Open Access which fosters wider collaboration and increased citations

- maximum visibility for your research: over $100 \mathrm{M}$ website views per year

At $\mathrm{BMC}$, research is always in progress.

Learn more biomedcentral.com/submissions 